\title{
Encoding and Retrieval in a CA1 Microcircuit Model of the Hippocampus
}

\author{
Vassilis Cutsuridis $^{1, \star}$, Stuart Cobb $^{2}$, and Bruce P. Graham ${ }^{1}$ \\ ${ }^{1}$ Department of Computing Science and Mathematics, University of Stirling, \\ Stirling, FK9 4LA, U.K. \\ $\{$ vcu, b.graham\}@cs.stir.ac.uk \\ ${ }^{2}$ Division of Neuroscience and Biomedical Systems, University of Glasgow, Glasgow, \\ G12 8QQ, U.K. \\ s.cobb@bio.gla.ac.uk
}

\begin{abstract}
Recent years have witnessed a dramatic accumulation of knowledge about the morphological, physiological and molecular characteristics, as well as connectivity and synaptic properties of neurons in the mammalian hippocampus. Despite these advances, very little insight has been gained into the computational function of the different neuronal classes; in particular, the role of the various inhibitory interneurons in encoding and retrieval of information remains elusive. Mathematical and computational models of microcircuits play an instrumental role in exploring microcircuit functions and facilitate the dissection of operations performed by diverse inhibitory interneurons. A model of the CA1 microcircuitry is presented using biophysical representations of its major cell types: pyramidal, basket, axo-axonic, bistratified and oriens lacunosummoleculare cells. Computer simulations explore the biophysical mechanisms by which encoding and retrieval of spatio-temporal input patterns are achieved by the CA1 microcircuitry. The model proposes functional roles for the different classes of inhibitory interneurons in the encoding and retrieval cycles.
\end{abstract}

Keywords: Hippocampus, CA1, microcircuit, computer model, pyramidal cell, basket cell, bistratified cell, axo-axonic cell, OLM cell, STDP.

\section{Introduction}

The hippocampus has been studied extensively, yielding a wealth of data on network architecture, cell types, the anatomy and membrane properties of pyramidal cells and interneurons, and synaptic plasticity [1]. It contains principal excitatory neurons (pyramidal (P) cells in CA3 and CA1 and granule cells (GC) in the DG) and a large variety of inhibitory interneurons 2 . Its basic functional role is hypothesized to be the intermediate-term storage of declarative memories 3 .

Many computational models have been advanced over the years trying to understand how memories are stored and retrieved in the hippocampus [12, [14, [23], 21], 6], [5], 9].

\footnotetext{
^ Corresponding author.

V. Kurkova, R. Neruda, and J. Koutnik (Eds.): ICANN 2008, LNCS 5164, pp. 238-247 2008.

(C) Springer-Verlag Berlin Heidelberg 2008
} 
A conceptual model of how GABAergic interneurons might provide the control structures necessary for phasing storage and recall in the hippocampus has been proposed recently [15. Building on this idea, we construct a functional model of the CA1 microcircuit, including a number of different neuronal types (pyramidal (P) cells, basket (B) cells, bistratified (BS) cells, axo-axonic (AA) cells and oriens-laconosum-moleculare (OLM) cells) and their specific roles in storage and recall. The recall performance of the model is tested against different levels of pattern loading and input presentation period.

\section{Materials and Methods}

\subsection{Model Architecture and Properties}

Figure 1 illustrates the simulated microcircuit model of the CA1 network. The model consists of 100 pyramidal (P) cells, 2 basket (B) cells, 1 bistratified (BS) cell, 1 axo-axonic (AA; chandelier) cell and 18 oriens lacunosum moleculare (OLM) cells. The cell percentages matched those found in rat hippocampus [4. All simulations were performed using NEURON [8] running on a PC under Windows XP. The morphology of each cell in the model was adapted from experimental studies 24], 25. The biophysical properties of each cell were adapted from cell types reported in the literature [16], [17, [19], [18].

Pyramidal Cells. Each P cell was modeled as 15 anatomical compartments. Membrane properties included a calcium pump and buffering mechanism, a calcium activated mAHP potassium current, an LVA L-type $\mathrm{Ca}^{2+}$ current, an HVA L-type $\mathrm{Ca}^{2+}$ current, an MVA R-type $\mathrm{Ca}^{2+}$ current, an HVA T-type $\mathrm{Ca}^{2+}$ current, an $\mathrm{h}$ current, an $\mathrm{HH}$ current that includes both a sodium and a delayed rectifier current, a slow $\mathrm{Ca}^{2+}$ - dependent potassium current, a slow non-inactivating potassium channel with $\mathrm{HH}$ style kinetics and a $\mathrm{K}^{+}$A current [16], 17]. Less than $1 \%$ recurrent connections between pyramidal cells in the network was assumed [26].

Each pyramidal cell received nine somatic synaptic inhibition contacts from the population of basket cells [27, mid-dendritic excitation from CA3, distal apical excitation from the entorhinal cortex (EC), proximal excitation from other pyramidal cells in the network (recurrent collaterals) [1, eight axonic synaptic inhibition contacts from the population of chandelier cells [27, 28, six middendritic synaptic inhibition contacts from the bistratified cells population 27 . and two distal synaptic inhibition contacts from each OLM cell.

Axo-Axonic Cells. Each AA cell was modeled with 17 compartments. Membrane properties included a leak conductance, a sodium current, a fast delayed rectifier $\mathrm{K}^{+}$current, an A-type $\mathrm{K}^{+}$current, $\mathrm{L}$ - and $\mathrm{N}$-type $\mathrm{Ca}^{2+}$ currents, a $\mathrm{Ca}^{2+}$-dependent $\mathrm{K}^{+}$current and a $\mathrm{Ca}^{2+}$ - and voltage-dependent $\mathrm{K}^{+}$current [18. No recurrent connections between AA cells were assumed 22.

Axo-axonic cells received excitatory inputs from the EC perforant path to their SLM dendrites and excitatory inputs from the CA3 Schaffer collateral to their SR dendrites. In addition, the axo-axonic cells received inputs from active 


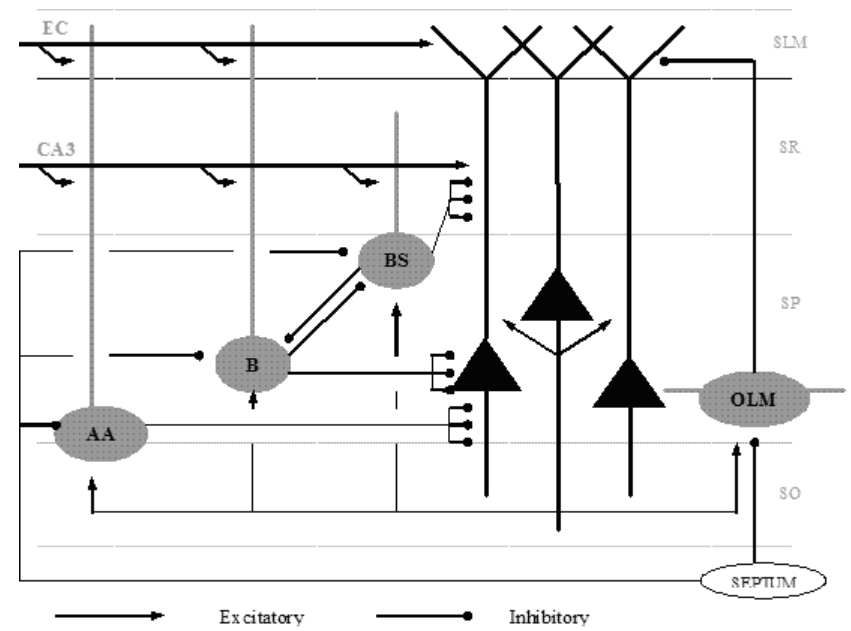

Fig. 1. CA1 microcircuit model of the hippocampus. EC: entorhinal cortex input; AA: axo-axonic cell; B: basket cell; BS: bistratified cell; OLM: oriens-lacunosum-moleculare cell; SO: stratum oriens; SP: stratum pyramidale; SR: stratum radiatum; SLM: stratum lacunosum-moleculare.

pyramidal cells in their SR medium and thick dendritic compartments as well as inhibitory input from the septum in their SO thick dendritic compartments [1].

Basket Cells. Each B cell was modeled with 17 compartments. Membrane properties included a leak conductance, a sodium current, a fast delayed rectifier $\mathrm{K}^{+}$current, an A-type $\mathrm{K}^{+}$current, L- and N-type $\mathrm{Ca}^{2+}$ currents, a $\mathrm{Ca}^{2+}$ dependent $\mathrm{K}^{+}$current and a $\mathrm{Ca}^{2+}$ - and voltage-dependent $\mathrm{K}^{+}$current 18 . Recurrent connections between all B cells and between all B and BS cells in the network were assumed [2].

All B cells received excitatory connections from the EC to their distal SLM dendrites, from the CA3 Schaffer collaterals to their medium SR dendrites and from active pyramidal cells to their medium and thick SR dendritic compartments and inhibitory connections from neighboring B and BS cells in their soma and from the medial septum in their SO thick dendritic compartments.

Bistratified Cells. Each BS cell was modeled with 13-compartments. Membrane properties included the same ionic currents as the B and AA cells. Recurrent connectivity between all BS and between BS and B cells in the network was assumed [2. All BS cells received excitatory connections from the CA3 Schaffer collaterals in their medium SR dendritic compartments and from the active pyramidal cells in their thick SO dendritic compartments and inhibitory connections from the medial septum in their thick SO dendritic compartments and from neighboring B and BS cells in their somas. 

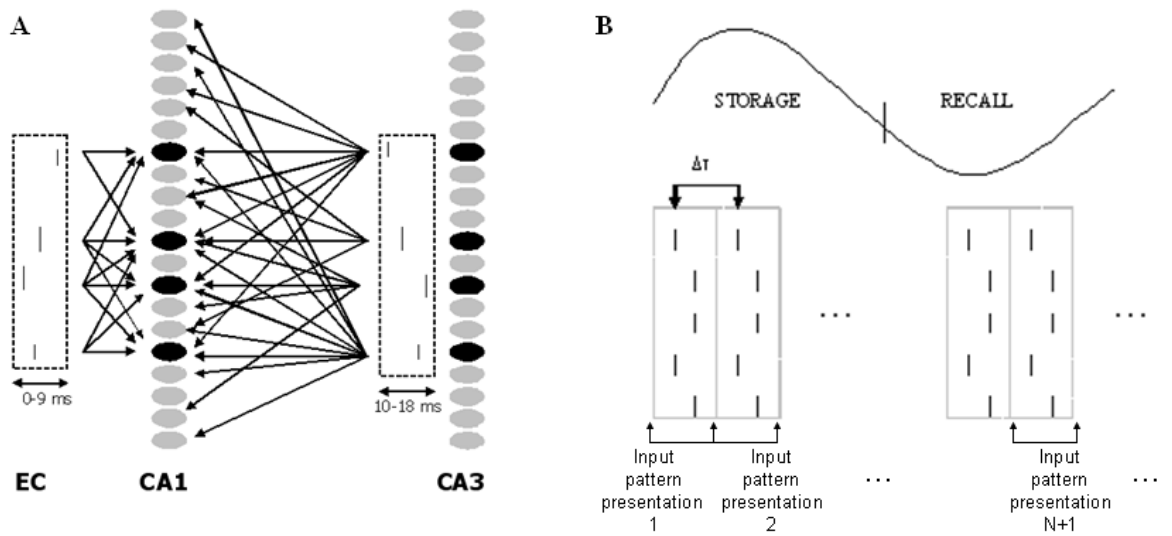

Fig. 2. (A) Model inputs to CA1 microcircuit. EC: entorhinal cortex input. The input arrives asynchronously in CA1 LM P cell dendrites (not shown) between 0-9 ms. A randomly selected subset of $\mathrm{P}$ cells receive the EC input. The CA3 input arrives asynchronously in CA1 SR P cell dendrites between $10-18 \mathrm{~ms}$ (that is, $10 \mathrm{~ms}$ after the EC input). All $\mathrm{P}$ cells non-selectively receive the CA3 input. (B) Input pattern presentation in the model. An input pattern was defined as the spatio-temporal sequence of asynchronously arriving spikes to $\mathrm{P}$ cells. The presentation of an input pattern is repeated every $\Delta \tau(5 \mathrm{~ms}, 7 \mathrm{~ms}, 8 \mathrm{~ms}, 10 \mathrm{~ms}, 11 \mathrm{~ms})$ continuously throughout the encoding and retrieval sub-cycles of the theta rhythm.

OLM Cells. Each OLM cell was modeled as a reduced 4-compartment model [19, which included a sodium $(\mathrm{Na}+)$ current, a delayed rectifier $\mathrm{K}^{+}$current, an A-type $\mathrm{K}^{+}$current and an h-current. No recurrent connections were assumed between OLM cells.

Each OLM cell received excitatory connections from the active pyramidal cells in their basal dendrites as well as inhibitory connections from the medial septum in their soma.

Model Inputs. Inputs to CA1 came from the medial septum (MS), entorhinal cortex (EC) and CA3 Schaffer collaterals. All P cells received the CA3 input, whereas a randomly selected subset of $\mathrm{P}$ cells received the EC layer III input (see figure $2 \mathrm{~A}$ ). All B, AA and BS in the network received the CA3 input, whereas only the AA and $\mathrm{B}$ cells received the EC input. The conduction latency of the EC-layer III input to CA1 LM dendrites is less than $9 \mathrm{~ms}$ (ranging between 5-8 ms), whereas the conduction latency of EC-layer II input to CA1 radiatum dendrites via the di/tri-synaptic path is greater than $9 \mathrm{~ms}$ (ranging between $12-18 \mathrm{~ms})[13$.

In the model, an input pattern was defined as the spatio-temporal sequence of asynchronously arriving spikes to corresponding $\mathrm{P}$ cells. The size of the input pattern was equal to the percentage of $\mathrm{P}$ cells that received the EC input. Both EC and CA3 input patterns were presented to P cell apical LM and medial SR 
dendrites respectively at various repeated time window shifts (i.e. each input was repeatedly presented with a period of $5 \mathrm{~ms}, 7 \mathrm{~ms}, 8 \mathrm{~ms}, 10 \mathrm{~ms}$, or $11 \mathrm{~ms}$ ) (see figure 2B). MS input provided GABA-A inhibition to all INs in the model (strongest to B and AA). MS input was phasic at theta rhythm and was on for $70 \mathrm{~ms}$ during the retrieval phase.

Synaptic Properties. In the model, AMPA, NMDA, GABA-A and GABA-B synapses were included. GABA-A were present in all strata, whereas GABA$B$ were present in medium and distal SR and SLM dendrites. AMPA synapses were present in strata LM (EC connections) and radiatum (CA3 connections), whereas NMDA were present only in stratum radiatum (CA3 connections).

Synaptic Plasticity. A local spike-timing dependent plasticity (STDP) learning rule was applied at medial SR AMPA synapses on P cells [22]. Pre-synaptic spike times were compared with the maximal postsynaptic voltage response at a synapse. If the interval is positive (a pre-synaptic spike arrives before the post-synaptic neuron response), then the synapse is potentiated by increasing the maximum AMPA conductance. If the interval is negative, the synapse is depressed by reducing the AMPA conductance.

Network Training and Testing. During encoding the maximum synaptic conductances of the SR AMPA synapses were allowed to change according to the learning rule explained above. During retrieval, the conductances from the last time window of input presentation were kept fixed throughout the entire retrieval cycle.

\section{Results}

In the model, we make two important assumptions, which are supported by experimental evidence: (1) Encoding and retrieval are two functionally independent sub-cycles of theta rhythm [7, and (2) During the storage sub-cycle the pyramidal cells that receive the EC input (i.e. the input pattern) do not fire action potentials [10, 11, 29] and hence the stored pattern does not "leak" out from the CA1.

\subsection{Encoding Cycle}

Previous modelling studies emphasized the role of a feedforward association of the incoming EC and CA3 inputs as the means of storing patterns in the CA1 P cells 31. However, recent experimental evidence [13] has shown that in $\mathrm{P}$ cells of CA1 the conduction latency of the EC-layer III input to their LM dendrites is less than $9 \mathrm{~ms}$ (ranging between 5-8 ms), whereas the conduction latency of EClayer II input to their radiatum dendrites via the di/tri-synaptic path is greater than $9 \mathrm{~ms}$ (ranging between 12-18 ms). That means that forward association of the EC- and CA3-inputs is not feasible, given that the information to be stored is 
contained in the coincident activity of cells in layers II and III of EC. Therefore, a different mechanism is required to associate the two inputs.

During the simulated storage cycle of the theta, we propose the following: an EC input pattern arrives to the apical SLM dendrites of the B, AA and P cells at time $\mathrm{t}_{i}$, whereas an indirect $\mathrm{CA} 3$ input pattern via the $\mathrm{di} /$ trisynaptic loop arrives to the medium $\mathrm{SR}$ dendrites of the $\mathrm{B}, \mathrm{AA}, \mathrm{BS}$ and $\mathrm{P}$ cells at time $\mathrm{t}_{i}+\delta t(\delta t>9 \mathrm{~ms})[13)$. In the $\mathrm{B}$ and AA cells, the EC input is strong enough to induce an action potential in their soma. Furthermore, the GABAergic cell population of the medial septum is minimally active and therefore transmits the least amount of inhibition to the CA1 inhibitory interneurons. Having the least amount of inhibition impinging on them, the CA1 inhibitory cells are free to do several things: First, the axo-axonic and basket cells exert tight inhibitory control on the axons and somas of the pyramidal cells, thus preventing them from firing during the storage cycle [10. Second, the basket cells exert powerful inhibitory control to neighbouring basket cells and to bistratified cells, which prevents the later from firing during the storage cycle. As mentioned earlier, the bistratified cells are 180 degrees out-of-phase with the basket and axo-axonic cells and hence not active during the storage cycle [11].

The CA3 input to $\mathrm{P}$ cells provides the contextual information, whereas the EC input to $\mathrm{P}$ cells provides the sensory information, because place cells in CA1 were found to be maintained solely by direct input from EC and CA1 [20. Since there is no topography in CA1 [26], during the storage cycle, $20 \%$ of the $\mathrm{P}$ cells in the network are randomly selected to receive the EC input pattern in their apical SLM dendrites (see figure 2). The summed postsynaptic potentials (PSP) generated in the SLM dendrites are attenuated on their way to the soma and axon 32, where they are finally "stopped" by the B and AA cell inhibition. Due to the strong B and AA cell inhibition on their soma and axon, non-specific hyperpolarizing h-activated cation channels are activated, which send a rebound back-propagating post-synaptic response (BPPR) towards the SR and SLM dendrites. In the model, to induce the BPPR, the conductance of the $\mathrm{I}_{h}$ current is increased 10-fold in the proximal SR dendrites ( $\mathrm{g}_{\text {hprox }}=0.0005$ $\mathrm{mS} / \mathrm{cm}^{2}$ ) and 20-fold in the medium and distal SR dendrites $\left(\mathrm{g}_{\text {hmed,dist }}=0.001\right.$ $\left.\mathrm{mS} / \mathrm{cm}^{2}\right)$ compared to the soma $\left(\mathrm{g}_{\text {hsoma }}=0.00005 \mathrm{mS} / \mathrm{cm}^{2}\right)$ 30. In contrast to the EC input, all $\mathrm{P}$ cells in the network are activated by the CA3 input in their medial SR dendrites. Careful timing between the incoming CA3 Schaffer collateral spike, the EC spike and the BPPR will induce potentiation (LTP) or depression (LTD) via a local STDP rule 22] applied in the medium SR dendrites of the pyramidal cells.

\subsection{Retrieval Cycle}

The retrieval cycle begins as the GABAergic cells of the septum approach maximum activity. Because of this septal input, the basket and axo-axonic cells are inhibited, releasing pyramidal cells, bistratified cells and OLM cells from inhibition. Pyramidal cells may now fire more easily, thus allowing previously learned patterns to be recalled. During the retrieval cycle, the CA3 Schaffer collateral 
input plays the role of a cueing mechanism: If the CA3 input excited a pyramidal cell during this time, any synapses that were strengthened during the storage cycle will be activated, recalling the memory. Because the CA3 input is directed to all $\mathrm{P}$ cells, which potentially activates unwanted $\mathrm{P}$ cells and hence spurious memories are recalled, the role of the bistratified cells is to ensure that these spurious cells will be silenced by broadcasting a non-specific inhibitory signal to all $\mathrm{P}$ cells in the network.

In our model during recall the entorhinal cortical input provides a weak background excitation to the CA1 region that aids the recall process, causing depolarized cells to fire. However, this excitation can potentially give rise to unwanted or similar memories. In our model, $\mathrm{P}$ cells after being released by the basket and axo-axonic cell inhibition excite the OLM cells. This excitation was assumed strong enough to overcome the OLM septal inhibition. In return, the OLM cells strongly inhibit the distal SLM dendrites of the P cells 2], where the direct entorhinal input arrives, thus preventing unwanted or similar memories from being recalled.

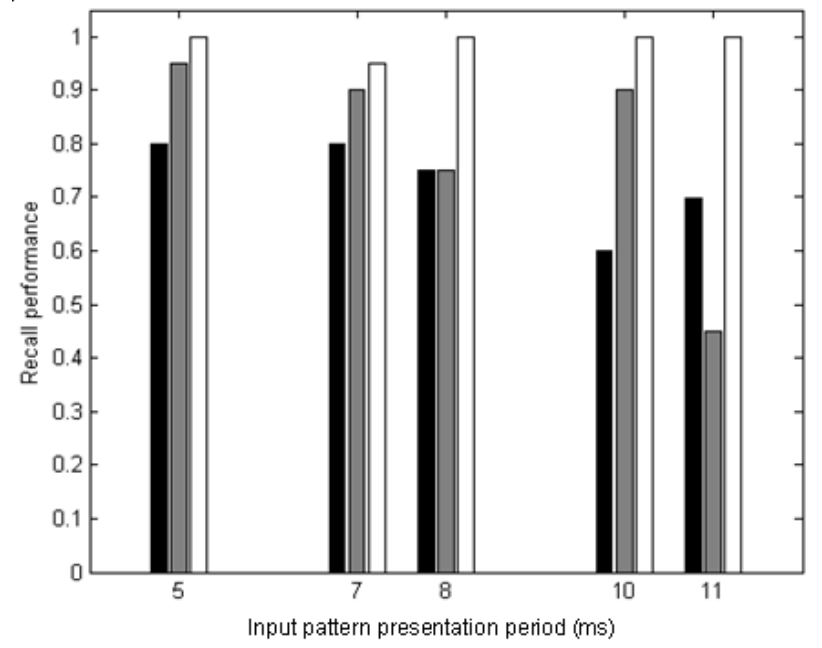

Fig. 3. Normalized recall performance during the 'many-trials' learning paradigm as a function of input pattern loading $(10 \%, 50 \%$ and $75 \%)$ and input pattern presentation period (a pattern is repeatedly presented every $5 \mathrm{~ms}, 7 \mathrm{~ms}, 8 \mathrm{~ms}, 10 \mathrm{~ms}, 11 \mathrm{~ms}$ ). White bars: $75 \%$ pattern loading; Grey bars: $50 \%$ pattern loading; Black bars: $10 \%$ pattern loading.

\subsection{Recall Performance}

Twenty percent of all $\mathrm{P}$ cells in the network received the EC input pattern (EC input pattern: a group of twenty spikes arriving asynchronously within a time window of 0-9ms (see figure 2B)) in their SLM dendrites, whereas all P cells 
received the CA3 input pattern (CA3 input pattern: the same group of twenty asynchronous spikes delayed by at least $9 \mathrm{~ms}$ ) arrive in their SR dendrites. As we said previously, both the EC and CA3 input patterns were continuously presented with a period $\Delta \tau(\Delta \tau=5-11 \mathrm{~ms})$ throughout the encoding and retrieval cycles of the theta rhythm. To estimate the recall performance of our network, we counted the fraction of cells belonging to the stored pattern that were active during the retrieval cycle.

Figure 3 depicts the model's recall performance for a particular input pattern. Different time shifts between pattern presentations during encoding and retrieval were tried $(\Delta \tau=$ every $5 \mathrm{~ms}, 7 \mathrm{~ms}, 8 \mathrm{~ms}, 10 \mathrm{~ms}$ or $11 \mathrm{~ms})$, and different levels of cue pattern loading $(10 \%, 50 \%$ and $75 \%$ of EC input pattern vector is presented to $\mathrm{P}$ cells) were used during recall. When $75 \%$ of the EC input pattern was presented during recall (i.e. $75 \%$ pattern loading), the recall performance was nearly perfect $(100 \%)$ regardless of input presentation period with the exception at $7 \mathrm{~ms}(95 \%)$. At $50 \%$ and $10 \%$ pattern loading, the recall performance dropped by $5 \%$ and $20 \%$ respectively when the input presentation period was $5 \mathrm{~ms}$. At larger input presentation periods, the recall performance degraded progressively for both $50 \%$ and $10 \%$ pattern loadings reaching a minimum of $45 \%$ and $70 \%$ respectively at $11 \mathrm{~ms}$.

\section{Conclusion}

A detailed model of the CA1 microcircuit has been presented. The model proposes functional roles for a variety of CA1 cells in the encoding and retrieval of memories in the hippocampus. The performance of the model is tested against different levels of pattern cueing during recall and different input pattern presentation time shifts during learning. These initial tests indicate that this circuitry can successfully store and recall patterns of information within a theta cycle. The quality of storage does depend on the temporal pattern presentation sequence, as recall performance drops when the time between presentations is increased and only a small cue is used during recall. Much work remains to be done to further explore the temporal constraints on this process and to assess more widely the capacity of this network to operate as an associative memory.

Acknowledgement. This work was funded by an EPSRC project grant to B. Graham and S. Cobb.

\section{References}

[1] Andersen, P., Morris, R., Amaral, D., Bliss, T., O'Keefe, J.: The hippocampus book. Oxford University press, Oxford (2007)

[2] Freund, T.F., Buzsaki, G.: Interneurons of the hippocampus. Hippocampus 6, 347-470 (1996)

[3] Eichenbaum, H., Dunchenko, P., Wood, E., Shapiro, M., Tanila, H.: The hippocampus, memory and place cells: is it spatial memory or a memory of space? Neuron 23(2), 209-226 (1999) 
[4] Baude, A., Bleasdale, C., Dalezios, Y., Somogyi, P., Klausberger, T.: Immunoreactivity for the GABAA receptor $\alpha 1$ subunit, somatostatin and connexion 36 distinguishes axoaxonic, basket and bistratified interneurons of the rat hippocampus. Cerebral Cortex 17(9), 2094-2107 (2007)

[5] Cutsuridis, V., Hunter, R., Cobb, S., Graham, B.P.: Storage and Recall in the CA1 Microcircuit of the Hippocampus: A Biophysical Model. In: Sixteenth Annual Computational Neuroscience Meeting CNS 2007, Toronto, Canada, July 8th 12th, 2007, vol. 8 (Suppl 2), p. 33. BMC Neuroscience (2007)

[6] Graham, B.P., Cutsuridis, V.: Dynamical Information Processing in the CA1 Microcircuit of the Hippocampus. In: Computational Modeling in behavioral neuroscience: Closing the gap between neurophysiology and behavior. Psychology Press, Taylor and Francis Group, London (December 2008) (to be published)

[7] Hasselmo, M., Bodelon, C., Wyble, B.: A proposed function of the hippocampal theta rhythm: separate phases of encoding and retrieval of prior learning. Neural. Comput. 14, 793-817 (2002)

[8] Hines, M.L., Carnevale, T.: The NEURON simulation environment. Neural. Comput. 9, 1179-1209 (1997)

[9] Hunter, R., Cutsuridis, V., Cobb, S., Graham, B.: Improving Associative Memory in a Model Network of Two-Compartment Spiking Neurons. In: Fourth Annual Scottish Neuroscience Group Meeting, University of Edinburgh (August 31, 2007)

[10] Klausberger, T., Magill, P.J., Marton, L.F., David, J., Roberts, B., Cobden, P.M., Buzsaki, G., Somogyi, P.: Brain-state- and cell-type-specific firing of hippocampal interneurons in vivo. Nature 421, 844-848 (2003)

[11] Klausberger, T., Marton, L.F., Baude, A., Roberts, J.D., Magill, P.J., Somogyi, P.: Spike timing of dendrite-targeting bistratified cells during hippocampal network oscillations in vivo. Nat. Neurosci. 7(1), 41-47 (2004)

[12] Kunec, S., Hasselmo, M.E., Kopell, N.: Encoding and retrieval in the CA3 region of the hippocampus: a model of theta-phase separation. J. Neurophysiol. 94(1), 70-82 (2005)

[13] Leung, L.S., Roth, L., Canning, K.J.: Entorhinal inputs to hippocampal CA1 and dentate gyrus in the rat: a current-source-density study. J. Neurophys. 73(6), 2392-2403 (1995)

[14] Menschik, E.D., Finkel, L.H.: Neuromodulatory control of hippocampal function: towards a model of Alzheimer's disease. Artificial Intelligence in Medicine 13, 99-121 (1998)

[15] Paulsen, O., Moser, E.: A model of hippocampal memory encoding and retrieval: GABAergic control of synaptic plasticity. TINS 21, 273-279 (1998)

[16] Poirazzi, P., Brannon, T., Mel, B.W.: Arithmetic of subthreshold synaptic summation in a model CA1 pyramidal cell. Neuron 37, 977-987 (2003a)

[17] Poirazzi, P., Brannon, T., Mel, B.W.: Pyramidal neuron as a 2-layer neural network. Neuron 37, 989-999 (2003)

[18] Santhakumar, V., Aradi, I., Soltetz, I.: Role of mossy fiber sprouting and mossy cell loss in hyperexcitability: a network model of the dentate gyrus incorporating cells types and axonal topography. J. Neurophysiol. 93, 437-453 (2005)

[19] Saraga, F., Wu, C.P., Zhang, L., Skinner, F.K.: Active dendrites and spike propagation in multicompartmental models of oriens-lacunosum/moleculare hippocampal interneurons. J. Physiol. 552(3), 673-689 (2003)

[20] Brun, V.H., Otnass, M.K., Molden, S., Steffenach, H.A., Witter, M.P., Moser, M.B., Moser, E.I.: Place cells and place recognition maintained by direct entorhinal-hippocampal circuitry. Science 296, 2243-2246 (2002) 
[21] Sommer, F.T., Wennekers, T.: Associative memory in networks of spiking neurons. Neural Networks 14, 825-834 (2001)

[22] Song, S., Miller, K., Abbott, L.: Competitive "hebbian" learning through spiketiming-dependent synaptic plasticity. Nat. Neurosci. 3, 919-926 (2000)

[23] Wallestein, G.V., Hasselmo, M.E.: GABArgic modulation of hippocampal population activity: sequence learning, place field development and the phase precession effect. J. Neurophys. 78, 393-408 (1997)

[24] Megias, M., Emri, Z.S., Freund, T.F., Gulyas, A.I.: Total number and distribution of inhibitory and excitatory synapses on hippocampal CA1 pyramidal cells. Neuroscience 102(3), 527-540 (2001)

[25] Gulyas, A.I., Megias, M., Emri, Z., Freund, T.F.: Total number and ration of excitatory and inhibitory synapses converging onto single interneurons of different types in the CA1 area of the rat hippocampus. J. Neurosci. 19(22), 10082-10097 (1999)

[26] Amaral, D., Lavenex, P.: Hippocampal neuroanatomy. In: Andersen, P., Morris, R., Amaral, D., Bliss, T., O'Keefe, J. (eds.) The Hippocampus Book, pp. 37-114. Oxford University press, Oxford (2007)

[27] Buhl, E.H., Halasy, K., Somogyi, P.: Diverse sources of hippocampal unitary inhibitory postsynaptic potentials and the number of synaptic release sites. Nature 368, 823-828 (1994a)

[28] Buhl, E.H., Han, Z.S., Lorinczi, Z., Stezhka, V.V., Kapnup, S.V., Somogyi, P.: Physiological properties of anatomically identified axo-axonic cells in the rat hippocampus. J. Neurophys. 71(4), 1289-1307 (1994b)

[29] Somogyi, P., Klausberger, T.: Defined types of cortical interneurons structure space and spike timing in the hippocampus. J. Physiol. 562(1), 9-26 (2005)

[30] Migliore, M., Hoffman, D.A., Magee, J.C., Johnston, D.: Role of an A-type K ${ }^{+}$ conductance in the back-propagation of action potentials in the dendrites of hippocampal pyramidal neurons. J. Comp. Neurosci. 7, 5-15 (1999)

[31] O'Reilly, R.C., McClelland, J.L.: Hippocampal conjunctive encoding, storage, and recall: avoiding a trade-off. Hippocampus 4(6), 661-682 (1994)

[32] Stuart, G., Spruston, N.: Determinants of voltage attenuation in neocortical pyramidal neuron dendrites. J. Neurosci. 18(10), 3501-3510 (1998)

[33] Bi, G.Q., Poo, M.M.: Synaptic modifications in cultured hippocampal neurons: dependence on spike timing, synaptic strength and postsynaptic cell type. J. Neurosci. 18, 10464-10472 (1998) 